\title{
Detection of infiltrating mast cells using a modified toluidine blue staining
}

\begin{abstract}
Mast cells are part of the immune system and characteristically contain histamine- and heparin-rich basophilic granules. While these cells are usually associated with allergy and anaphylaxis, they also promote wound healing and angiogenesis and confer protection against pathogens. The presence of these cells is sometimes indicative of a poor prognosis, especially in skin cancer, pancreatic cancer, and lymphoma. Toluidine blue staining of acid-fast granules is an established method for the identification and quantification of mast cells. Generating detailed information on the location of mast cells within tissues is problematic using this technique and often requires serial sections from adjacent tissue to be separately stained with hematoxylin and eosin (H\&E). Staining serial sections is not always possible, particularly if the sample is very small or rare. In such cases, a method of simultaneously identifying and localizing mast cells in a tissue would be advantageous. Toluidine blue and $H \& E$ are not commonly combined because $H \& E$ includes repetitive washes in water, which may affect the efficacy of the aqueous-soluble toluidine blue. We have developed and tested a novel staining technique that integrates toluidine blue between hematoxylin and eosin in one simple procedure. This protocol works on both frozen and formalin-fixed, paraffinembedded tissue and readily allows for the identification of purple-stained mast cells against a clean H\&E background. This facilitates a more accurate localization and proper counting of mast cells in normal and affected tissue
\end{abstract}

Keyword: Hematoxylin and eosin; Inflamed intestine; Mast cells; Skin; Toluidine blue 